\title{
El desarrollo de la teoría del desarrollo
}

E1 desarrolio del capitalismo estuvo en el centro del pensamiento económico durante el siglo posterior a la publicación, en 1776, de La riqueza de las naciones, de Adam Smith. Esa era la preocupación fundamental cle la economía política clásica. Durante el último cuarto del siglo x.x y hasta airedecor de 1950, esta preocupación, y la propia economía politica, fucron desplazadas de la corriente principal clel pensamiento económico. Se impusieron la teoría económica neoclásica, con su énfasis en la conducta de los productores y consumidores inclividuales en mercados perfectos o imperfectos, y las teorias que trataban de explicar la inestabilidad cíclica del capitalismo.

El tema del desarrollo sólo comenzó a retornar al pensamiento económico en los años 50 , pero ahora refericlo al problema econó. mico de los países que no habían llegado a industrializarse hacia esa época. El desarrollo económico de los paises subelesarrollados, que es lo que realmente tenemos en mente cuando hablamos ahora de la teoría del desarrollo, es un tema muy reciente en la evolución del pensamiento económico. Dificilmente algún libro pulblicaclo antes de 1950 contenia la palabra clesarrollo en su titulo, las universidades no ofrecian cursos sobre la materia, no existian institutos especiales de desarrollo, ni expertos en este campo, La asistencia técnica, tal como la conocemos ahora, sólo se convirtió en una operación sustancial con el programa del Punto IV del Presidente Truman y con el programa cle asistencia técnica de la O.N.U.

Esta primera reunión interregional de las cuatro asociaciones que representan a cientos de instituciones y miles de investigadores dedicados a los estudios sobre el desarrollo en Africa, Asia, Europa y América Latina, refleja la enorme importancia que los estudios y actividades sobre el desarrollo han adquiriclo a lo largo de las últimas clos clécadas y meclia. ${ }^{1}$

1 Primera Reunión Interregional sobre In Investigación, la Comunicación y la Enseñanza del Desarrollo, realizada en el Instituto de Estudios del Desarrollo, Universidad de Sussex, clel 12 al 16 de setiembre de 1976 , y organizada conjuntamente por el Instituto y el Centro dle Estuclios del Desarrolio de la OECD. Este articulo es una versión revisala del cliscurso pronunciado por el autor en la sesión inarigural tle dicha reunión. 
Pero el auge de que ha gozado el tópico del desarrollo está en total contraste con la crisis del proceso de desarrollo. Una de las consecuencias ha sido una reevaluación crítica del pensamiento y de las políticas. En un momento en que se proponen nuevas politicas, estrategias y modelos de desarrollo, puede ser útil detenerse y contemplar la evolución del pensamiento sobre la materia. La interacción entre la teoría y la práctica del desarrollo a lo largo de las dos últimas décadas puede proporcionar importantes lecciones para el futuro.

Examinaré la evolución del pensamiento sobre el desarrollo en los últimos 25 años desde tres perspectivas principales: la naturaleza de las sociedades que constituyen el "objeto" de la teoría y su contexto internacional; la naturaleza de la teoría económica de que se disponía cuando el desarrollo se transformó en un tema fundamental en la década de 1950; y las reacciones en el pensamiento sobre el desarrollo causadas por las consecuencias del propio proceso de desarrollo.

Antes de emprender esta difícil tarea - una especie de sociología del conocimiento del pensamiento sobre el desarrollo- me gustaría recalcar que este ejercicio no está basado en una investigación sistemática, sino más bien en. la experiencia personal; que esta experiencia es esencialmente la de América Latina -y por lo tanto de la abrumadora presencia hegemónica de los Estados Unidos; y que es la experiencia de un economista.

Empezaré por referirme a algunas de las principales características estructurales de las economias subdesarrolladas alrededor de 1950, como también a algunos cambios básicos que se estaban produciendo internamente y en el contexto internacional.

La mayor parte de los paises subdesarrollados aún eran colonias en ese tiempo, algunos habian llegado a ser independientes recientemente, y otros - la mayoria de los paises latinoamericanos- habían sido independientes por mís de un siglo. Todos estaban estrechamente vinculados económica, política y culturalmente a alguno de los principales paises industrializados, al cual exportaban productos primarios y excedentes financieros, y de los cuales importaban manufacturas, recursos humanos, inversiones, tecnologia, instituciones, ideas, valores, $y$, en gencral, cultura.

La magnitud del sectior que "consumía" esas importaciones -el llamado sector moderno- dependia básicamente de tres factores: a) el tamaño del excedente total generado por el sector exportador; b) la proporción de este excedente que los grupos dirigentes locales lograban retener para el consumo o la inversión (mientras más invertian localmente más se expandia su base productiva); y c) el grado hasta el cual los grupos dirigentes explotaban el resto de la 
sociedad, sea mediante la preservación parcial y la explotación de las instituciones y las culturas locales preexistentes, como a través de su destrucción parcial a fin de generar un suministro abundante de tierra y de mano de obra barata. Como consecuencia de esto, la economía, Ia sociedad, la politica y la cultura nacionales eran altamente heterogéneas; subsistían varios modos de organización socioeconómica, politica y cultural pero su interacción se realizaba esencialmente bajo relaciones capitalistas de comercio y/o de producción.

Los efectos de la Gran Depresión, la Segunda Guema Mundial, la descolonización y la Guerra Fría, contribuyeron en muchos paises a un profundo cambio en el balance de poder dentro de las ciases dirigentes. Los grupos relacionados más estrechamentie con los intereses exportadores e importadores tradicionales se debilitaron, y se fortalecieron nuevos sectores de la clase media: profesionales, pequeños empresarios, inmigrantes con experiencia y habilidad empresarial, industriales -allí donde previamente se había desarrollado alguna industria-, artesanos, funcionarios, algunos sectores de empleados y obreros. El objetivo básico de estos nuevos grupos sociales era la promoción de la industrialización como un medio para generalizar las pautas de consumo y los estilos de vida que sus élites habían adquiriclo a través de la importación de los estilos de vida de los países industriales (el "efecto de demostración.", como fue llamado entonces). Para hacer" posible esto, los nuevos grupos sociales tenían que afirmar su control sobre el sector externo para poder captar una mayor proporción del excedente, necesaria para financiar un consu. mo y una inversión crecientes. De estla manera, el Estado llegó a ser el instrumento fundamental de una nueva política destinada a la reproducción local de las características de los países capitalistas maduros; industrialización, modernización agrícola, infraestructura, servicios sociales, etc. Los países que tenían todas estas cosas cran los países desarrollado; los que no Jas tenían era subdesarrollados, y el desarrollo era el proceso de transición de una situación a la otra.

Esta conceptualización del desarrolo fue intensamente promovida desde el exterior, particularmente por los Estados Unidos -el nuevo centro motriz del sistema capitalista-como un importante instrumento de politica exterior en el contexto de la Guerra Fría. El apoyo externo a las clases dirigentes nacionales contra cualquicr desafío de la izquierda llegó a ser un objetivo extremadamente importante en el campo capitalista". Por otra parte, las clases dirigentes nacio-

2 Este no fuc solamente el caso en el mundo subdesarrollado, sino más aún el de Europa; los principales instrumentos fueron la NATO, el plan Marshal y la integración económica europea. 
nales en los países subdesarrollados veian a los Estados Unidos y a Europa Occidental como guardianes de sus intereses -a quienes recurrir en caso de necesidad-, y como modelos sociopoliticos y económicos, a quienes se debía imitar. El clesarrollo del capitalismo industrial moderno en esos países llegó a ser, por lo tanto, la meta común a largo plazo de las clases dirigentes de esos paises y de los Estados Unidos. Esto contribuyó frecuentemente a una aceleración de la desintegración de los imperios coloniales europeos y a un mayor fortalecimiento de los Estados Uniclos como el superpcder capitalista.

Veamos ahora rápidamente el pensamiento de que se disponía al* rededor de 1950, para ayudar a Ios estadistas, expertos y asesores de esa época a formular sus ideas acerca de las políticas más adecuadas para el clesarrollo de los países subdesarrollados.

Existia por supuesto, la economía politica, el locus classicus del análisis del surgimiento $y$ expansión del capitalismo industrial en Gran. Bretaña. Pero los escritos de los padres fundadores de la economía política se consideraban superados por el progreso científico de la disciplina, y por lo tanto habian sido relegados al papel de piezas de museo en la historia del pensamiento económico. El principal aporte analítico de la economía politica clásica - las relaciones entre la expansión del modo de producción capitalista y la operación del mercado con los cambios en la estructura de las clases sociales y la consiguiente redistribución y uso del poder, en el contexto histórico de la expansión imperialista del capitalismo industrialfue precisamente lo que habia sido expurgado de la economia politica y reemplazado por la teoría económica "positiva". El cuerpo de pensamiento más amplio y relevante heredado de la economía política clásica -el marxismo- había sido suprimido en todas partes: en el mundo capitalista por el McCarthysmo, en el socialista por el stalinismo. En nombre del progreso científico y de la Guerra Fría, la clinámica sociopolitica del capitalismo había sido exorcisada de la economía, imponiéndose dos escuelas principales de economía "pura": Ia teoria económica neoclásica, incluyendo la teoria cle las ventajas comparativas en el comercio internacional, y la macroeconomía keynesiana, con sus variantes de corto y largo plazo.

Estas corrientes teóricas correspondian intimamente a las necesidades y caracteristicas del capitalismo avanzado: maximizar la utilidad de empresas y consumidores individuales en los mercados nacioles e internacionales, por una parte, $y$ por la otra, evitar la inestabilidad ciclica con políticas de pleno empleo en el corto plazo y de crecimiento en el largo plazo. Pero para los paises subdesarrollados, con las características antes descritas, las politicas derivadas de estas corrientes de pensamiento económico equivalían a un drástico pro- 
grama de transformaciones sociopoliticas, económicas y culturales, puesto que muy pocos de los supuestos subyacentes en la economia neoclásica y keynesiana estaban presentes. $O$, para ser más precisos; estos supuestos sólo correspondian a un segmento de la realidad, aquél más estrechamente vinculacio con el sector exportador $y$ las grandes ciudades. La moneda no era un medio universal de intercambio, era usada en transacciones urbanas y hasta cierto punto en las urbano-rurales, pero raramente dentro (o entre) las comuniclades rurales. Con la excepción de algunas de las actividades de exportación, y del sector urbano, difícilmente podia hablarse de un mercado de trabajo, ya que mucha gente permanecía vinculada a comunidades agrarias y otras instituciones rurales. Las empresas y los empresarios modernos, de tipo capitalistas, eran fundamentalmente extranjeros y se encontraban principalmente en el sector exportador y en el mercado y sólo en pequeño grado en un sector manufacturero muy precario. El capital social básico - caminos, energía, comunicaciones, ferrocarriles, puertos, etc.- también se concentraba en torno a las actividades de exportación y de las ciuclades principales; éstas estaban de hecho en mejor y más fácil contacto con los centros metropolitanos que con el interior de su propio pais. La educación estaba restringida a una pequeña élite urbana. Las instituciones financieras, excepto algunas sucursales de bancos extranjeros eran escasas y de capacidad limitada. El aparato estatal estaba restringido en cuanto a la extensión geográfica y la variedad de sus operaciones $y^{\prime}$ tenía una base impositiva muy circunscrita y altamente inestable, especialmente en el sector externo. ${ }^{3}$

Si la teoría no correspondía a la realidad, tanto peor para esta última: la realidad debería ser cambiacla a fin de hacerla corresponfler a los supuestos de las teorías económicas neoclásica y macrodináunica. Surgieron así dos lineas principales de pensamiento y de política. Por una parte, los representantes intelectuales del viejo orden, quienes sostenían que la especialización tradicional en las exportaciones primarias constituian el mejor "motor de crecimiento", siempre que los países industriales mantuvieran una alta tasa de crecimiento con pleno empleo. Los beneficios de la especialización y de las ventajas comparativas se transmitirian a otros sectores $y^{\prime}$ se extenderian al resto de la sociedad, de modo que se obtendria eventualmente el desarrollo. Esta línea de pensamiento contrariaba los intereses de los nuevos grupos sociales que estaban emergiendo de la

i Es obvio que este conjunto de caracteristicas estructurales, aunque siempre presente en todo el país subdesarollado, varia ampliamente en sus manifestaciones concretas de un caso a otro, de acuerdo con su peculiar experiencia formativa histórica. 
lucha contra el viejo orden: la alianza preexistente de las oligarquías locales y el imperialismo. Las nuevas burguesfas nacionales estaban tratando de obtener el control del Estado a fin de captar una mayor cuota del excedente generado por el sector exportador y utilizarlo para promover la industrialización y la modernización en general. Si bien por diferentes razones y en diferentes circunstancias a aquellas enfocadas por Keynes, la teoría macrodinámica proporcionó la racionalización para promover un papel activo del Estado y para considerar la acumulación de capital como la base del crecimiento económico. Los modelos de crecimiento -incluyendo los modelos de acumulación de capital usados en la planificación soviética- y la naturaleza operacional de la macroeconomía en términos de contabilidad social y análisis insumo-producto, constituyeron los instrumentos prácticos para la planificación (o más bien "programación", el eufemismo que se inventó en vista de la atmósfera de Guerra Fria).

Un conjunto de teorías que criticaban las doctrinas del libre comercio $y$ la especialización internacional y que atribuian el subdesarrollo a la falta de industrialización, proporcionaron la racionalización para establecer políticas proteccionistas, invertir en infraestructura e industrialización y adoptar la planificación (Prebish, Singer, Lewis, Mandelbaun, Rosenstein, Rodan, Nurkse y otros —ninguno de ellos- hecho interesante, de origen anglosajón). El contraste entre la realidad heterogénea que caracterizaba a los países subdesarrollados, descrita anteriormente, y los supuestos de la teoría neo-clásica conjuntamente con la imagen que proyectaban los paises industriales, proporcionaron el fundamento de las politicas de modernización y reforma institucional de la agricultura, la educación, el sistema tributario, la administración pública, el mercado de capitales, etc.

Una versión más radical de este programa para el desarrollo del capitalismo nacional, influida por el pensamiento marxista sobre la explotación interna e internacional, afloraba de vez en cuando, según las condiciones políticas. En este caso, se acentuaba especialmente la necesidacl de nacionalizar la inversión extranjera en el sector exportador y en. los servicios públicos, de ampliar el rol del Estado en la industrialización, de desarrollar el comercio con los páses socialistas, de llevar a cabo una drástica reforma agraria y de extender e intensificar la planificación estatal.

En América Latina, estas diversas líneas de pensamiento cristalizaron clurante los años 50 alrededor del trabajo de Raúl Prebisch y de un grupo de jóvenes economistas en la Comisión Económica para América Latina, de las Naciones Unidas. Esta institución llegó a ser una importante base de apoyo intelectual y técnico de los mo- 
vimientos $y$ gobiernos progresistas, nacionalistas y reformistas, a lo largo de la región, y el objeto de violentos ataques por parte de los gobiernos latinoamericanos más reaccionarios y del gobierno, los intereses económicos e importantes sectores académicos de los Estados Unidos.

Es fácil ahora, con la ventaja de una mayor perspectiva histórica, enumerar las debilidades y limitaciones de las nuevas políticas de desarrollo propuestas, y seguidas en mayor o menor medida, desde 1950. Pero en una época en que la alianza de los intereses sociopolíticos y económicos vinculados con los sectores exportadores primarios, tanto en los paises centrales como en los periféricos, era aún muy fuerte, y cuando cualquier amenaza a estos intereses era considerada automáticamente como un complot comunista y duramente reprimida, es conveniente recalcar que estos programas reformistas de carácter social demócrata y nacionalista eran considerados un desafío revolucionario al orden interno e internacional existente.

Todo esto cambió en forma bastante dramática a fines de los años 50, con la Revolución Cubana y la caídla de los dictadores Pérez Jiménez en Venezuela, Odría en Perú y Rojas Pinilla en Colombia, así como también con el cambio de la administración republicana de Eisenhower por la demócrata de Kennedy en los Estiados Unidos. Estos sucesos politicos agudizaron la crisis del viejo orden primario exportador, que no podía seguir subsistiendo por cuanto se había erosionado sustancialmente su base interna en América Latina, como señalé anteriormente, y también en el plano internacional, como se verá luego. Las alternativas históricas se perfilaban claramente: o bien la revolución socialista, el apoyo total a las nuevas fuerzas sociales que emergian e impulsaban las políticas de modernización e industrialización dentro de un modelo capitalista de desarrollo económico.

Se pensó que este programa económico, más la expansión de la educación, la modernización rural y la urbanización, promoverían la movilidad social y' la diversificación de la estructura social, ampliando en particular las clases medias y empresariales, la base social indispensable cle una sociedac, capitalistia moderna. La creación de sociedades burguesas modernas de este tipo facilitaría a su vez el desarrollo de la democracia política, como se practicaba en Europa Occidental $y$ en los Estados Unidos.

Las teorias económicas, sociológicas y politicas del desarrollo, que proporcionaban una versión. idealizada de los países que habían alcanzado la etapa del capitalismo industrial moderno, fueron transformadas en programas de desarrollo capitalista en la periferia, en la ideología del clesarrollo. Las etapas del desarrollo económica; Un 
manifiesto no comunista, de Rostow, constituye probablemente la versión más extrema y explícita de la nueva idéología. La "Alianza para el Progreso", la respuesta de las nuevas élites industriales de los Estados Unidos y América Latina a la Revolución Cubana y al establecimiento de un estado socialista en las Américas, era la expresión práctica y operacional de un programa masivo de modernización capitalista.

Cómo se mencionó antes, el contexto de la Guerra Fria, y en especial la Revolución Cubana, junto con los cambios en la estructura del poder interno en América Latina fueron factores fundamentales en la promoción del desarrollo $y$ los programas de modernización en América Latina. Pero además influían otras fuerzas, de naturaleza más económica y global, que apoyaban. estos esfuerzos. El surgimiento de Ios Estados Unidos como la superpotencia en el campo capitalista, con su vasta dotación de recursos naturales y su sistema industrial extremadamente dinámico y expansivo, estaba comenzando a cambiär la naturaleza del orden económico intemacional construido por Gran Bretaña y Europa Occidental en el Siglo XIX. Después de la Segunda Guerra Mundial, la gran expansión de la inversión americana en el exterior se concentró en las manufacturas y en los servicios comerciales y financieros relacionados con este' sector, antes que en los productos primarios, excepto el petróleo. Institucionalmente, este es el período de la fenomenal expansión de las corporaciones transnacionales. La Alianza para el Progreso y en términos más generales, el desarrollo, representan por lo tanto la alianza para el progreso de las nuevas élites industriales transnacionales del centro y las élites modernizantes de los paises periféricos.

Durante algunos años, a principios de la década de 1960, Ios esfuerzos para el desarrollo nacional e internacional parecian estar en el camino hacia una aceleración de la industrialización, la modernización, la urbanización, la movilidad social, la descolonización y la democracia política en los países que por entonces eran llamados "subdesarrollados", y que luego fueron rebautizados como paises "en vias de desarroilo". Las palabras de Marx: "el país más desarrollado solo muestra a los menos desarrollados la imagen de su propio futuro" parecian estar en vías de ser verificadas por la historia.

Pero el historial del desarrollo durante los años 60 y principios de los 70 resultó más ambivalente que el optimismo alentado por el logro de tasas de crecimiento económico, industrialización y urbanización sin precedentes. Si bien se suponía que el crecimiento económico acelerado aumentaría las oportunidades de empleo, pronto se hizo evidente que el subempleo y el desempleo, particularmente en las áreas urbanas, estaba creciendo en forma dramática, creando 
candentes problemas de pobreza urbana. En parte como consecuencia de este fenómeno, pero también debido a la gran concentración de 1a riqueza, el ingreso y la educación superior en manos de pequeñas élites empresariales y profesionales, el crecimiento económico acentuó la desigualdad del ingreso. Si bien la expansión yi diversificaciớn industrial habia Ilegado bastante lejos en muchos paises, los beneficios asociados históricamente con la industrialización en los países desarrollados no se hacian presente. En vez de contribuir a la diversificación de la estructura de las exportaciones, los productos primarios continuaron predominando. En vez de contribuir a la formación y fortalecimiento de la clase empresarial local, las subsidiarias de las corporaciones transnacionales acapararon las actividades industriales más dinámicas y las empresas de mayor tamaño. Con el creciente control extranjero de la industria se introdujeron aceleradamente innovaciones de alta y creciente intensidad de capital que contribuyeron a incrementar el consumo conspicuo, el desperdicio del capital existente, el aumento de las importaciones y otros gastos en moneda extranjera. Ante la imposibilidad politica de llevar a cabo reformas estructurales en las áreas rurales, li modemización de la agricultura aumentó el rendimiento y la productividad por hombre en los grandes predios, generando rentas adicionales para la clase terratenientc, y contribuyendo a la desintegración y al estancamiento de las comunidades rurales y de los pequeños propietarios, acelerando la emigración rural. El mismo fenómeno de "subsidiarización", modernización y expansión de las empresas mayores, y la consiguiente desorganización, desplazamiento y estagnación de las firmas nacionales más pequeñas, con la resultante polarización social, puede apreciarse en mayor o menor grado en cada rama de la actividad económica: comercio, construcción, finanzas, transporte, medios de comunicación, etc., tanto en el sector público como en el privado.

El creciente desempleo urbano y la polarización social condujeron a una acentuación de las políticas "sociales": nuevos intentos de introducir impuestos progresivos, crecientes gastos gubernamentales en educación, vivienda $y$ servicios de salud, programas especiales en apoyo de las clases urbanas más pobres, politicas regionales para áreas postergadas. Dadas las situaciones estructurales $\mathrm{y}$ los procesos subyacentes que estas políticas intentaban remediar, sus efectos fueron, en el mejor de los casos, insignificantes y en el peor, una contribución a las tendencias negativas amiba enumeradas.

Los desequilibrios macroeconómicos de las economias embarcadas en estos programas de desarrollo llegaron a ser cada vez más dramáticos durante la última década. Los gobiernos habian estaclo expandiendo fuertemente sus gastos y actividades, mientras sus ingresos con- 
tinuaban derivándose en gran medida' de un sector externo relativamente estancado y altamente inestable con la consiguiente crisis fiscal. Del nuismo modo, los pagos externos en cuenta corriente y de capital se elevaron rápidamente con el aumento cle las inversiones, el consumo, la propiedad extranjera y la deuda externa pública y privada, mientras lá estructura exportadora permanecía esencialmente inalterada y con escaso dinamismo. El crecimiento de la población y el ingreso urbano frecuentemente sobrepasaron el aumento de la producción rural comercializable. Estos y otros desequilibrios alimentaron la inflación interna y las crisis de balanzas de pagos, aumentando aún más el endeudamiento externo. La crisis económica agudizó las tensiones sociales y políticas, y ello se tradujo con frecuencia en la instalación de gobiernos autoritarios, que han procedido a aplicar severas políticas deflacionarias, adoptando medidas fuertemente represivas contra las clases populares. El desarrollo, en vez de aliviar represisiones y de facilitar la democracia política, de hecho agravó la polarización sociocconómica y cultural y acentuó el conflicto político.

Enfrentado con esta situación inesperada, el pensamiento sobre el desarrollo tomó dos direcciones opuestlas. Una argumentaba que esta no era sino la consecuencia inevitable de la transición hacia el desarrollo en el futuro. La otra elaboró una crítica radical, sugiriendo que el desarrollo capitalista en la periferia no reproduciria el camino que siguió en el pasado en los países ahora desarrollados, y que su actual crisis no sería superada insistiendo en políticas de desarrollo del mismo tipo. La primera aproximación es obviamente aquella que aún siguen la mayoría de los expertos en todo el mundo. Pero si bien el pensamiento sobre el desarrollo en Ios círculos académicos, especialmente en los países desarrollados, contlinúa basado en gran me. dida en el paradigma de la modernización; como consecuencia de la dinámica interna - -o más bien de la estadística interna- del mundo académico, el pensamiento sobre el desarrollo en América Latina comenzó a cambiar radicalmente a mediados de los años 60, particularmente entre algunos científicos sociales que habian estado íntimamente comprometidos con las políticas y la planificación del desarroIlo.

Para comenzar, resulıaba cada vez más claro que había existido una reificación de la economía en el pensamiento sobre el desarrollo, llegándose prácticamente a la exclusión de las otras ciencias sociales. Pero si este era claramente el caso, la solución - que había sido practicada hasta cierto punto- no consistía en colocar las otras disciplinas al lado de la ciencia económica - una suerte de enfoque interdisciplinario por agregación. El problema es mucho más profundo $y^{\prime}$ tiene que ver con el paradigma funcionalista y estático de 
todas las ciencias sociales modernas: el estudio del funcionamiento económico, social y político de una sociedad nacional, atendidas las condiciones histórico-estructurales del moderno capitalismo urbanoindustrial. El problema del desarrollo, por el contrario, comenzó a percibirse cada vez más como el estudio del cambio sociocultural producido por la expansión del modo capitalista de producción dentro de formaciones sociales semi y/o precapitalistas. Se comenzó a advertir además que el propio capitalismo moderno estaba sufriendo cambios significativos bajo la influencia del nuevo rol central desempeñado por el sector de los intereses transnacionales, en simbiosis con el Estado, en el proceso de acumulación de capital, de innovación tecnológica y de manipulación de lá demanda en un escala global. El estudio del desarrollo, por lo tanto, requiere necesariamente una mejor comprensión de la dinámica del capitalismo contemporáneo, tanto en. su núcleo central como en sus periferias, asíi como en sus relaciones recíprocas. Por último, pero muy importante, porque es un hecho históricamente inédito, en sus relaciones con el campo socialista.

En otras palabras, se hizo evidente que la unidad de análisis del desarrollo no podía ser el estado-nación en. forma aislada. Aunque es obvio que debemos concentrarnos en el estudio del país en el que estamos interesados, su peculiar proceso histórico de desarrollo nacional debe ser colocado en el contexto de la evolución del capitalismo global, y de sus manifestacioncs locales o internas. Estas han constituido típicamente un conjunto crucial cle factores determinantes que a largo plazo han provocado profundos procesos de transformaciones estructurales. El establecimiento de las colonias, la lucha contra los colonizadores, la descolonización, el desarrollo de las exportaciones de productos primarios, la inversión extranjera en las manufacturas locales, los efectos de las guerras mundiales y de las crisis, las transferencias de instituciones extranjeras y de la cultura en general, son todos fenómenos que juegan un rol central en la evolución histórica de los países subdesarrollados.

Aunque hayan sucedido en épocas diferentes y tomen diversas formas y aspectos, estos y otros fenómenos similares constituyen el legado histórico común de la dependencia en nuestros países y las reacciones que desencadenan dependen por supuesto, y búsicamente, de la naturaleza de las sociedades precxistentes y de su respuesta a las manifestaciones internas de los estímulos externos. Por lo lianto, entre los países subdesarrollados hay elementos comunes así como grandes diferencias. Una perspectiva global o eurocéntrica tiende a subrayar la homogeneidad; la perspectiva nacional, por el contrario, subraya 
la heterogeneidad y la singularidad. Ambas perspectivas son sesgadás y deben complementarse mutuamente.

El creciente cuerpo de literatura sobre la evolución histórica dè países y regiones en América Latina, Africa y Asia, en el contexto đel desarrollo del capitalismo global, está arrojando una nueva luz sobré la naturaleza real del proceso de clesarrollo. La historia económicà y social ha recibido de hecho un gran estímulo en la última década como consecuencia de las preguntas planteadas por la crisis del desáarollo, y la historia comparada ha sido un ejercicio particularmentè útil para una mejor comprensión de los procesos de formación dè situaciones contemporáneas. Este no ha sido un ejercicio puramentè acalémico, la historia por su propio provecho, sino un instrumentó esencial para la interpretación de los sucesos actuales.

La reincorporación de la dimensión histórica dentro del pensamiento sobre el desarrollo tiene otros efectos altamente positivos. Eh lo que respecta a la economía, ha tenido la saludable consecuencià de forzar láa aclaptación del aparato analítico, conceptual y' teórico de la disciplina económica a la presente realidad histórica, en vee de tó contrario. La teoría convencional micro y macro económica está siendo reformulada en un esfuerzo para abstraer variables y relacionés relevantest y un esfuerzo correspondiente se está realizando para elaborar nuevas categorias operacionales en términos de marcos de re=ferencia estadísticos apropiados.

La recuperación de la dimensión histórica ha puesto también én claro que la especialización disciplinaria de las ciencias sociales bajo el paradigma funcionalista, las ha hecho inherentemente incapaces de captar la naturaleza del proceso de clesarrollo. Esto no pretende negar su utilidad para el análisis de situaciones parciales concretas, donde los supuestos y las realidades no están tan alejadas, pero llegan inevitablemente a constituir ideologia cuando son usadas para interpretar el desarrollo de la sociedad como un todo.

El marco analítico de la economía política clásica, y particularmente el marxismo, si bien no es enteramente satisfactorio al menos va en la dirección requerida para analizar el desarrollo: globalismo y totalidad. Pero al mismo tiempo, para ser verdaderamente útil, este marco analítico requiere especifidad histórica, esto es, el análisis de las características estructurales de una sociedacl en un lugar y tiempo determinado, puesto que estos son los determinantes del funcionamiento $y$ desarrollo de tal sociedad. En otras palabras, el modo de operación de una economía capitalista, aun si presumimos que las leyes básicas del desarrollo capitalista no cambian, varia bajo diferentes arreglos institucionales y tradiciones culturales. El desarrollo capitalista no es, como nos quisieran hacer creer los modelos de cre- 
cimiento macrodinámicos, un proceso expansivo de tipo mecánico, clonde todo el futuro estra determinado por un conjunto inmutable cle condiciones iniciales.

Por el contrario, como lor indica la experiencia de cualquiera que haya participado en la formulación y aplicación de políticas de desartollo, el desarrollo capitalista tiene lugar dentro y en interacción con formaciones precapitalistas y/o con formaciones capitalistas anteriores. Su naturaleza expansiva requiere una profunda reorganización cle la sociedad, con nuevas fuerzas sociales que reúnan el poder Y la energia necesarios para desafiar a los grupos dominantes existentes $y$ para apoderarse de las instituciones que regulan la generacion, apropiación y utilización del excedente económico. E1 crecimiento económico implica en la estructura social, una redistribución del pocler politico, transformaciones institucionales y culturales, y éste es un proceso dialéctico y conflictivo. No sólo debe haber cambios esuructurales e institucionales, sino que estos cambios se obtienen a través de lá confrontación. El Estado, el principal instrumento legitimado de la fuerza y el poder, es por ello normalmente el campo de batalla crucial de los diferentes grupos socialies.

En otras palabras, el Estado constituye un factor central en el proceso de desarrollo y es el instrumento político básico del cambio, particularmente en los países subdesarrollados donde es el medio fundamental para lograr una creciente participación en el excedente generado por el sector externo, y para su reasignación a los nuevos grupos sociales dominantes. La pobreza de las ciencias sociales, en particular de la economía, incluyendo en este cașo la economia politica clásica, en el tratamiento del rol del Estado, hạ sido sin cluda una de las mayores debilidades de la teoría del clesarrollo. No es sorprendente, por lo tanto, que haya llegado a ser una preocupación tan fundamental en años recientes.

El fracaso para apercibir el desarrollo como desarrollo capitalista, la ignorancia de la historia del desarrollo capitalista, tanto en el centro como en la periferia, la falta cle reconocimiento de las características peculiares del capitalismo contemporáneo, y la idcología de la modernización, son algunas de las razones fundamentales de la sorpresa con que los especialistas en clesarrollo han recibido los rẹsultados del desarrollo: crecimiento económico con desempleo creciente, polarización y desigualdad en aumento, nuevas formas de dependencia, regímenes autoritarios. Las anteojeras ideológicas del paradigma de la modernizacion dirigen toda la atención hacia los aspectos positivos y ex post del desarrollo capitalista, lratando sus resultados finales -altos niveles de vida, moderada desigualclad, estilos de vida urbano-industriales, clemocracia politica- como medios para el desarro- 
Llo; Ia historia real del desarrollo capitalista puesta patas arriba. E1 desarrollo capitalista, como Schumpeter señaló tan acertadamente hace mucho tiempo, es un proceso de "destrucción creativa". Conviene tal vez recalcar que cuando Europa Occidental estaba siendo industrializada durante el Siglo XIX, una proporción sustancial de su población no sólo debió dejar el campo, sino que turo que emigrar a ultramar. Alrededor de 60 millones de personas dejaron Europa entre 1840 y 1920 , en tanto que su población total en 1900 era de solo unos 300 millones. Bajo las condiciones presentes de rápida expansión. mundial de un capitalismo oligopólico altamente dinámico, innovador $y$ de gran intensidad de capital, los efectos destructivos del desarrollo son particularmente severos, contribuyendo a acentuar las desigualdades y desniveles, tanto dentro como entre los países centrales y periféricos.

Dos décadas de crecimiento económico sin precedentes en la-economía capitalista también han tenido de hecho algunos efectos no anticipados sobre los mismos paises inclustriales, produciendo fuertes reacciones críticas: alineación, consumismo, desperdicio, concetración de poder, destrucción del medio ambiente, burocratización, pérdida de empleos debido a la transferencia de industrias al extranjero, etc. La depresión reciente, extrañamente unida a la inflacion, la crisis monetaria internacional, la incerticlumbre con respecto al futuro del crecimiento económico, la creciente ineficacia cle las politicas keynesianas, la relativa declinación del control sobre los recursos naturales del mundo y la creciente preocupación acerca de su disponibilidad, la amenaza del socialismo en Europa y los paises dependientes, son todos fenómenos que contribuyen a arrojar serias cludas acerea de si los así llamados paises desarrollados son realmente un modelo ideal de desarrollo.

Dichos paises también están enfrentando ahora profundos problemas de desarrollo; estos problemas no son sólo nacionales sino globales, no son sólo económicos sino sociales, culturales y políticos. Como consecuencia de ello, las preocupaciones de los científicos sociáles en los paises industrializados están comenzando a dirigirse otra vez hacia lo que constituia el tema central de la economia politica clásica en el Siglo XIX y del pensamiento sobre el desarrollo en los úlbimos años: el clesarrollo del capitalismo. 